\title{
THE ASTRO-D MISSION
}

\author{
Y. TANAKA \\ Institute of Space and Astronautical Science \\ 3-1-1 Yoshinodai, Sagamihara, Kanagawa-ken 229, Japan
}

\begin{abstract}
Astro-D, the fourth X-ray astronomy mission of ISAS following Ginga, is a high-throughput imaging and spectroscopic X-ray observatory, scheduled for launch in early 1993. It utilizes multilayer conical X-ray mirrors which provide a large effective area over a wide range energy range up to $12 \mathrm{keV}$. The focal plane instruments comprize a set of CCD cameras and Imaging gas scintillation proportional counters. Main features of Astro-D are described, and the important astrophysical objectives are discussed.
\end{abstract}

\section{Introduction}

The Institute of Space and Astronautical Science (ISAS) has launced three X-ray astronomy satellites so far. The first small X-ray astronomy satellite "Hakucho" was launched in 1979. "Tenma", carrying for the first time the large-area gas scintillation proportional counters, was launched four years later (1983). The third X-ray astronomy observatory "Ginga" launched in early 1987 carries a large-area (4000 $\mathrm{cm}^{2}$ ) proportional counter array jointly prepared by the Japanese and the U.K. groups, and is currently operational for its fourth year in orbit.

Astro-D is the fourth X-ray astronomy mission of ISAS, following Ginga. Astro$\mathrm{D}$ is designed to be a high-capability X-ray observatory from which a number of important astrophysical investigations can be carried out. The main features of Astro-D are given in Table I, and the schematic view is shown in Fig. 1. It will be equipped with multilayer thin-foil telescopes which provide a large effective area over a wide energy range from below $1 \mathrm{keV}$ up to $12 \mathrm{keV}$. It is capable of imaging the $\mathrm{X}$-ray sky with a spatial resolution comparable to that of the IPC of the Einstein Observatory. Two different types of detectors, imaging gas scintillation proportional counters and CCD cameras, are employed for the focal plane instruments. The CCDs will provide a spectral resolution comparable or superior to that of SSS of the Einstein Observatory but cover a much wider energy range. This is particularly powerful for the spectroscopic studies of X-ray sources, i.e. observations of the characteristic lines and absorption edges of elements from oxygen through iron. These feat ures characterize Astro-D to be the first high-throughput imaging and spectroscopic X-ray observatory covering a wide energy band over $10 \mathrm{keV}$.

Astro-D will be launched by the ISAS launch vehicle M-3S II into an approximately circular orbit of a $550-650 \mathrm{~km}$ altitude. The total weight is limited to 420 $\mathrm{kg}$. The spacecraft is three axis stabilized. The absolute pointing accuracy will be approximately 1 arcminute. However, the spacecraft stability and the accuracy of post facto attitude reconstruction is ecpected to be better than 0.2 arcminutes.

The key elements of Astro-D have been successfully tested during the PM phase, and activities for the flight hardware (FM) fabrication have started for the launch scheduled for early 1993 . 


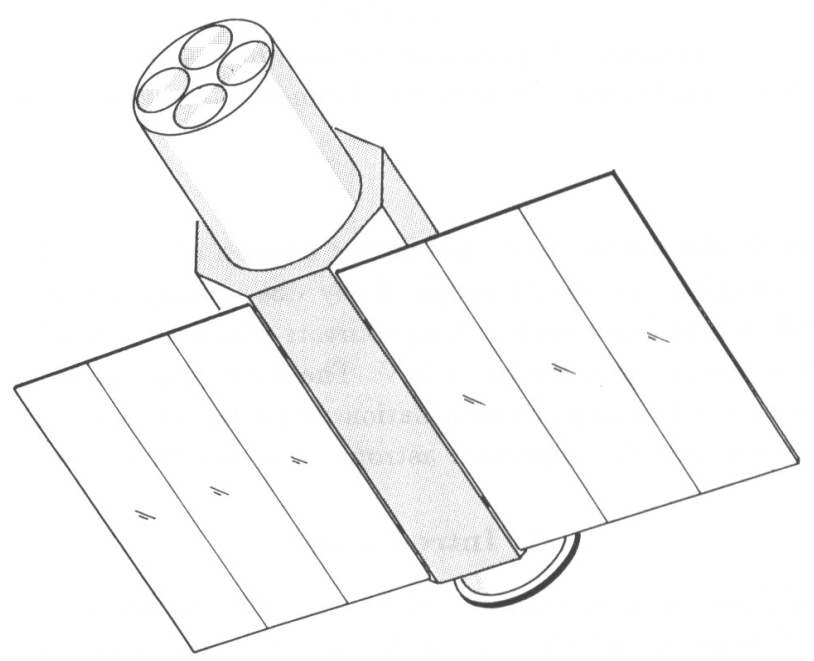

Fig. 1. Schematic view of Astro-D.

TABLE I

Main features of Astro-D

1. High-throughput over a wide energy range

Telescope: $\quad 4$ sets of multilayer thin-foil conical mirrors (collaboration with GSFC)

Effective area: $\sim 1300 \mathrm{~cm}^{2}$ at $1 \mathrm{keV}$ $\sim 600 \mathrm{~cm}^{2}$ at $6-7 \mathrm{keV}$

Focal length: $\quad 3.5 \mathrm{~m}$

Image size: $\quad \sim 2 \mathrm{~mm}$ half power diameter $(\mathrm{HPD})$

2. Imaging capability

Focal plane instruments:

2 sets of Imaging gas scintillation proportional counters

\& 2 sets of X-ray CCD cameras (jointly with MIT/Penn.State)

Field of view: $30 \times 30 \mathrm{arcmin}^{2}$

Point source resolution: $<1$ arcmin.

3. Good energy resolution

$\leq 8 \%(5.9 \mathrm{keV})$ with IGSPC

$\leq 2 \%(5.9 \mathrm{keV})$ with $\mathrm{CCD}$ (cooled to $\left.\sim-70^{\circ} \mathrm{C}\right)$ 


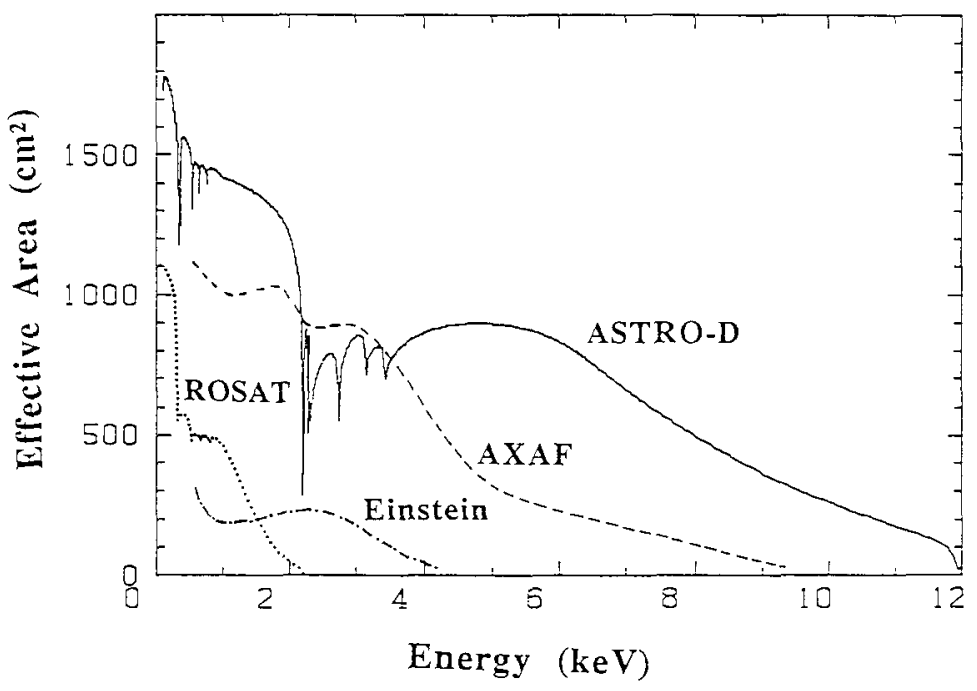

Fig. 2. On-axis effective area of four X-ray mirrors as a function of energy. Those of the Einstein Observatory, ROSAT and AXAF are also shown for comparison.

\section{X-Ray Telescopes}

The X-ray telescope consists of four sets of multilayer thin-foil conical mirrors. This technolgy is developed by P. Serlemitsos (1981). The mirror system of Astro-D is prepared by a collaboration between P. Serlemitsos and his collaborators at NASA Goddard Space Flight Center and the Nagoya University/ISAS group. An actual $\mathrm{X}$-ray telescope consists of two stages, each 120 layers of coaxially aligned thin-foil cones in an approximate Wolter type I configuration. The radii of the outermost and the innermost cones are $17.3 \mathrm{~cm}$ and $5.5 \mathrm{~cm}$, respectively, and each segment lenght is $10.9 \mathrm{~cm}$. For such a short segment length and a long focal length, the approximation of a praboloid or a hypaboloid by a cone is fairly good. However, the image size actually achieved at GSFC is approximately 2.5 arcminutes halfpower-diameter (HPD) at present due to several error sources, among which the shape error has been identified to be most important. An effort to improve the image quality for Astro-D is under way.

Although the image resolution is moderate, this technology can provide a large effective area over a wide range with a very light weight. Therefore, it is particularly advantageous for Astro-D which is severly weight limited. Astro-D carries four identical telescopes with a focal length of $3.5 \mathrm{~m}$. The total on-axis effective area for four sets of mirrors Astro-D as a function of X-ray energy is shown in Fig. 2, in comparison with those of the mirrors on board the Einstein Observatory, ROSAT and $A X A F$, respectively. 

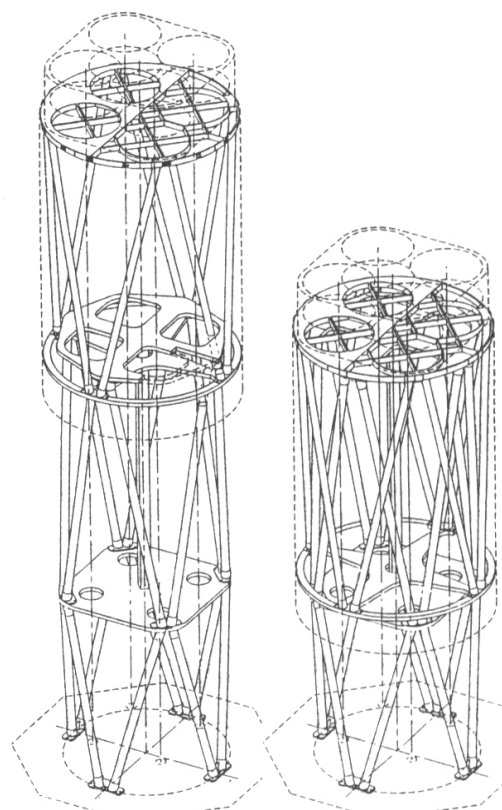

Fig. 3. Configurations of the optical bench during launch (top) and after extension (bottom).

\section{Optical Bench}

The nose fairing of the present ISAS launch vehicle, M-3S II, cannot accomodate an optical bench of a $3.5 \mathrm{~m}$ focal length. In order to achieve the required focal length, we employ an extensible optical bench which is folded short during the launch. It is extended in orbit to a $3.5 \mathrm{~m}$ focal length by means of a sliding mechanism, and latched precisely in position. Figure 3 shows schematically the configurations during launch and after fully extended, respectively. The optical bench is constructed by a truss structure composed of CFRP tubes in order to maintain the required accuracy against temperature changes. A prototype model of the extensible optical bench has been manufactured and verified. The entire CFRP surface will eventually be coated with aluminium in order to prevent outgassing.

\section{Focal plane instruments}

The focal plane instruments consist of two sets of IGSPC's and two sets of CCD cameras. These four detectors are placed at the foci of the four X-ray telescopes, respectively. These detectors will operate independently and simultaneously.

Each IGSPC is filled with Xe and sealed off with a thin berylium window. The IGSP's will cover the entire field of view of the telescope (larger than the coverage of the CCD camera). IGSPC has also a larger (and also less energy-dependent) detection efficiency for high energy photons above $5 \mathrm{keV}$ than the CCD camera.

The CCD cameras are prepared jointlsy by G. Ricker and his collaborators at 


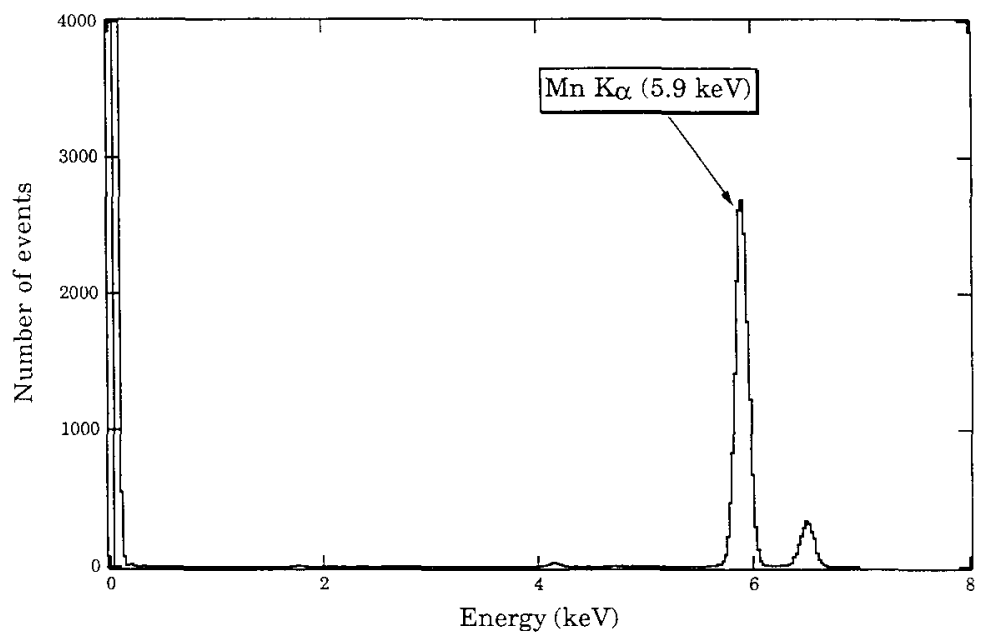

Fig. 4. Pulse-height spectrum of Mn-K lines obtained with a Lincoln CCD.

MIT and the Osaka University/ISAS team. Penn. State University group is also involved in the software development. A CCD camera head consists of the Lincoln Laboratory $840 \times 840$ hybrid CCD of front-side illumination (four $420 \times 420$ CCD chips abutted side by side, each read out by a separate preamplifier). The field of view coverage is $23 \times 23$ arcminutes $^{2}$. G. Ricker and his collaborators at MIT have been developing the X-ray CCD technology and demonstrated the excellent characteristics of the Lincoln lab. CCD's. An energy resolution of $\sim 2 \%$ for $5.9 \mathrm{keV}$ (Mn-K) photons has been achieved at a modest temperature of $\sim-70^{\circ} \mathrm{C}$. Figure 4 illustrates an example of the pulse-height spectrum of the Mn-K lines obtained with a Lincoln $\mathrm{CCD}$. The $\mathrm{K}$-alpha and $\mathrm{K}$-beta lines are distinctly separated from each other.

The CCD camera will be a powerful instrument of Astro-D in particular for the spectroscopic studies of the lines from oxygen through nickel. It is intended to use CCD's of $a \sim 60$ micron thick depletion layer, covering the entire energy range above $0.5 \mathrm{keV}$. The exptected detection efficiencies versus photon energy for IGSPC and CCD on board Astro-D are shown in Fig. 5.

Figure 6 shows the simulated energy spectra of a supernova remnant (Tycho's SNR) measured with a conventional proportional counter (PC), a gas scintillation proportional counter (GSPC), and a solid state detector (SSD) (CCD for Astro-D), respectively. IGSPC will resolve the characteristic line of each individual element unambiguously with a higher detection efficiency for high-energy X-rays. On the other hand, CCD can well resolve the K-alpha and K-beta lines of iron, and also enables measurement of the Doppler shift or broadening corresponding to a velocity of the order of $1000 \mathrm{~km} / \mathrm{sec}$ which is considered to be typical in X-ray sources. 


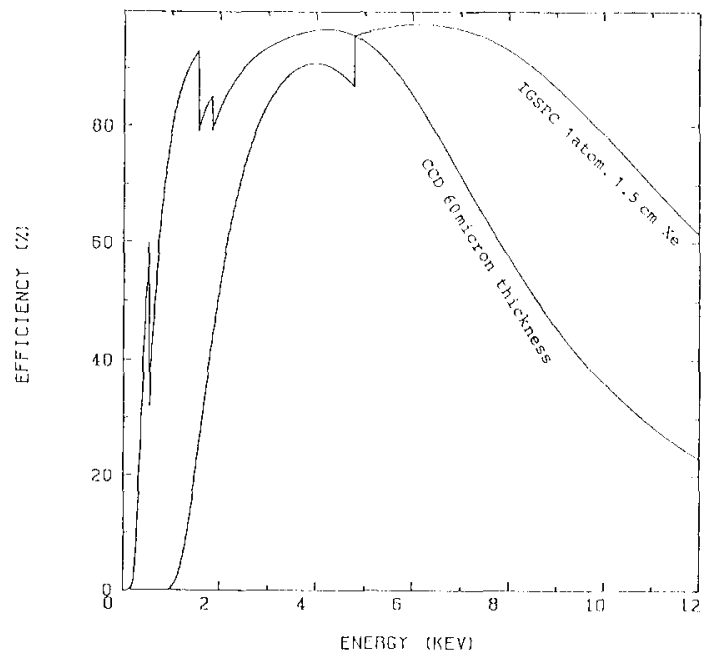

Fig. 5. Expected detection efficiencies for IGSPC and CCD.

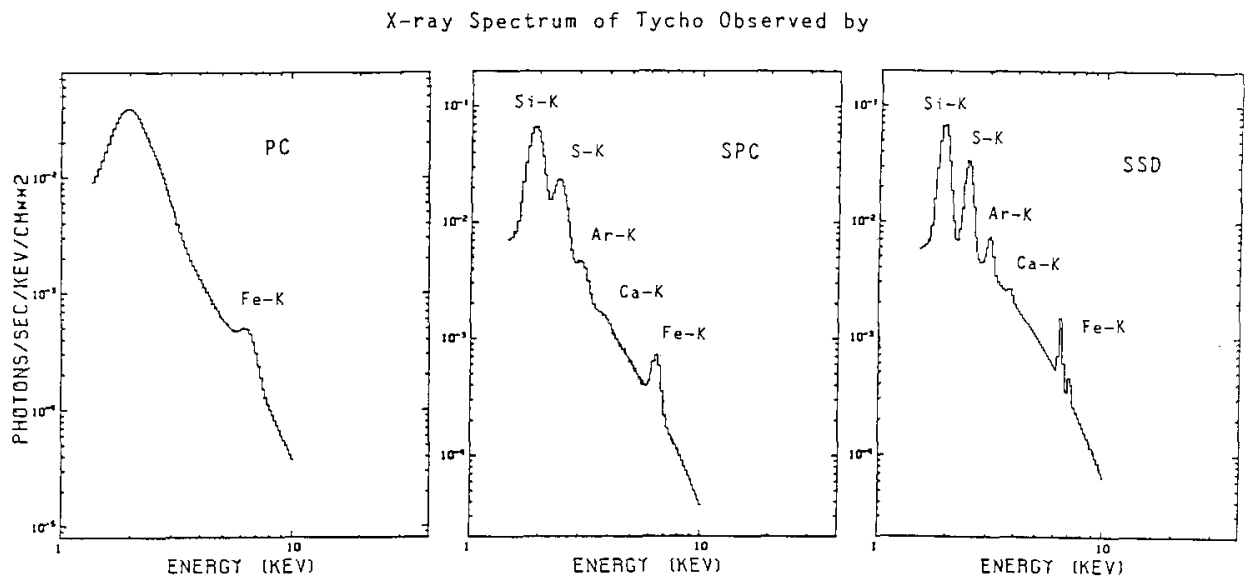

Fig. 6. Simulated spectra of Tycho's SNR measured with PC, GSPC and SSD, respectively.

\section{Scientific Objectives}

With the capabilities described above, a variety of important scientific objectives can be addressed. Table II lists these objectives to be investigated with Astro-D. Among others, we consider the problem of cosmic X-ray background (CXRB) to be one of the most important issues. The largest contributor to CXRB has been considered to be AGNs. However, thus far observed AGN spectra including many of those observed from Ginga are ualitatively different from that of CXRB. If discrete sources are to account for CXRB, Astro-D should be able to find new class of objects with much harder spectra than so-far known AGNs. 
TABLE II

Scientific objectives of Astro-D

* Active galactic nuclei

AGN's 10 times fainter than the Einstein limit

Spectra over a wide energy range

Detection of characteristic lines

* Cluster of galaxies

Temperature and abundance structure to $\mathrm{z} \sim 1$

Evolutionary effect

Sunyaev-Zel'dovich effect

* Cosmic X-ray background

Deeper than Einstein limit over a wide energy range

Spatial fluctuation

New population of galaxies, or diffuse?

* Galactic sources

Spectroscopy: Emission and absorption lines,

Absorption edges

Doppler shifts and broadening

Variability: Nature of QPO, flickering

Individual sources in the neighboring galaxies

* Supernova \& Supernova remnants

SN 1987 A

Spatial structure

Temperature and abundance structure

Search for neutron stars

* Emission along the galactic ridge

Spatial structure

Unidentified SNR's and/or new population sources?

\section{References}

Serlemitsos, P.J.: 1981, Broad Band X-Ray Telescope (BBXRT), X-Ray Astronomy in 1980 's, NASA TM 83848 , p. 441 